\section{In vitro optical performance of a new aberration-free intraocular lens}

D Madrid-Costa, J Ruiz-Alcocer, T Ferrer-Blasco, S García-Lázaro and R Montés-Micó
Optometry Research Group, Optics Department, University of Valencia, Valencia, Spain

Correspondence: D Madrid-Costa, Optometry Research Group, Optics Department, University of Valencia, C/ Dr Moliner 50, Burjassot, 46100 Valencia, Spain

Tel: +34 963544764

Fax: +34 963544764 .

E-mail: david.madrid@uv.es

Received: 21 January 2013 Accepted in revised form: 15 January 2014 Published online: 21 February 2014

\begin{abstract}
Purpose To assess the optical performance of the new EnVista intraocular lens (IOL). Materials and methods Four aspheric IOLs were evaluated; the new EnVista is one amoung them. This IOL, similarly to the Z-Flex HB and the Bi-Flex 1.8 667AB ones, has a neutral aspheric design, whereas the fourth IOL under test (AcrySof IQ IOL SN60WF) presents a negative spherical aberration (SA). The IOL's aberration patterns were measured in vitro, by setting them up on an optical bench. From these aberration-pattern data, the modulation transfer function (MTF), the average modulation values, and the points spread function (PSF) were calculated. Furthermore, in order to assess the potential optical quality that these IOLs would yield once they are implanted, an average cornealaberration pattern was juxtaposed to the in-vitro profiles and the same parameters were calculated again.

Results For the IOL-only scenario (ie, without including the corneal factor), it was the EnVista IOL, which is aberration-free that showed the higher MTF, PSF values. This was followed by the other two aberrationfree IOL models. However, when the effect of an average corneal pattern was also taken into consideration, the AcrySof IQ IOL SN60WF always outperformed the other neutral-asphericity IOLs.

Conclusions The in-vitro optical performance of the EnVista IOL was good, but it decreases substantially in a whole-eye scenario, when the wavefront profile of an average cornea is added. Other designs with different degrees of SA should be considered for this IOL in order to surpass these results.

Eye (2014) 28, 614-620; doi:10.1038/eye.2014.25;

published online 21 February 2014
\end{abstract}

\section{Introduction}

In order to improve their product offering, the specialized industry of intraocular lenses (IOLs) has launched periodic innovations into the market. One of the most important areas of innovation is related to wavefront technology. The introduction of this technology has highlighted the role of higherorder aberrations (HOAs), the spherical aberration (SA) being the most important one. Classic IOLs are spherical (consequently, with positive SA), whereas the latest models have aspheric designs. Within this aspheric-lens category, some of the IOLs have had its intrinsic SA wholly corrected (ie, they have no SA, which is why they are usually designated: 'aberration-free' IOLs), whereas other aspheric IOLs have been devised to compensate (totally or partially) the corneal SA (ie, the IOL itself has a negative SA), which is why they are often called 'aberrationcorrecting' IOLs.

At the same time, the materials from which the IOL is manufactured represent the other main field of innovation; in this respect, new materials are continuously being developed. Nowadays, the most widely implanted IOLs are made of hydrophobic acrylic materials, as these lenses are soft and foldable and, as a result, only a small incision is required for it to be inserted. These small incisions do not usually need sutures, thus allowing faster patient recovery times, minimizing residual astigmatism and providing better visual outcomes. $^{1-3}$

The aim of this study is to assess the optical performance of a novel hydrophobic acrylic IOL recently launched into the market. This new lens is the EnVista (Bausch and Lomb, Rochester, NY, USA). The optical performance of this new IOL was compared with three other commercially available IOLs. 


\section{Materials and methods}

\section{Aberration-free aspheric IOLs}

Among the four IOLs evaluated in this study, three of them look for attaining zero SA. The EnVista IOL is marketed as an aberration-free IOL. This lens is manufactured in hydrophobic acrylic material that incorporates a UV filter. This hydrophobic acrylic onepiece monofocal IOL has a total diameter of $12.5 \mathrm{~mm}$, with a $6.0 \mathrm{~mm}$ optical zone. The lens is available with an optical power that ranges from 0.0 to $34.0 \mathrm{D}$, in steps of $0.5 \mathrm{D}$ (within the $+10.0 \mathrm{D}$ to $+30.0 \mathrm{D}$ interval) or of $1 \mathrm{D}$ (within the 0.0 to $+9.0 \mathrm{D}$ and the +31.0 to $+34.0 \mathrm{D}$ intervals).

The other two aspheric aberration-free lenses under assessment were the Bi-flex 1.8 677AB (Medicontour, Geneva, Switzerland) and the Z-Flex HB 860FAB IOLs (Medicontour). The Bi-Flex 1.8 677AB is a one-piece IOL, manufactured from a combination of a hydrophilic and a hydrophobic copolymer. It includes a UV-absorber filter. The IOL's total diameter is $13.0 \mathrm{~mm}$, with a $6.0 \mathrm{~mm}$ optical zone. The lens is available with an optical power that ranges from 0.0 to $45.0 \mathrm{D}$, in steps of $0.5 \mathrm{D}$ (within the $+10.0 \mathrm{D}$ to $+30.0 \mathrm{D}$ interval) or $1 \mathrm{D}$ (within the 0.0 to $+9.0 \mathrm{D}$ and the +31.0 to $+45.0 \mathrm{D}$ intervals). Finally, as for the Z-flex HB 860FAB, it is a hydrophobic acrylic IOL that incorporates a blue filter. Its total diameter is $13.0 \mathrm{~mm}$, with a $6.00 \mathrm{~mm}$ optical zone. This lens is available with an optical power that ranges from -10.0 to $45.0 \mathrm{D}$, in steps of $0.5 \mathrm{D}$ (within the $+10.0 \mathrm{D}$ to +30.0 $\mathrm{D}$ interval) or $1 \mathrm{D}$ (within the -10.0 to $+9.0 \mathrm{D}$ and the +31.0 to +45.0 D intervals).

\section{Aberration-correcting IOL}

The AcrySof IQ IOL SN60WF (Alcon Laboratories, Fort Worth, TX, USA) IOL features an aspheric modification to the posterior optic surface. The aim of this design is to provide a negative amount of SA $(-0.20 \mu \mathrm{m}$ for a $6-\mathrm{mm}$ pupil), thus compensating part of the positive SA that the eye shows after crystalline lens' extraction. The lens is a foldable single-piece hydrophobic acrylic IOL that incorporates both a UV and a blue-blocker filter (yellow tint). The optical zone is $6.0 \mathrm{~mm}$ in diameter, whereas the lens' total width is $13.0 \mathrm{~mm}$. The optical power of AcrySof IQ IOL SN60WF ranges from +6.0 to $+30.0 \mathrm{D}$, in $0.5 \mathrm{D}$ steps.

\section{Image quality assessment}

To assess the IOLs' image quality we used the NIMO instrument (LAMBDA-X, Nivelles, Belgium), which includes an optical bench, together with its software version 4.5.15. Figure 1 shows a schematic layout of the

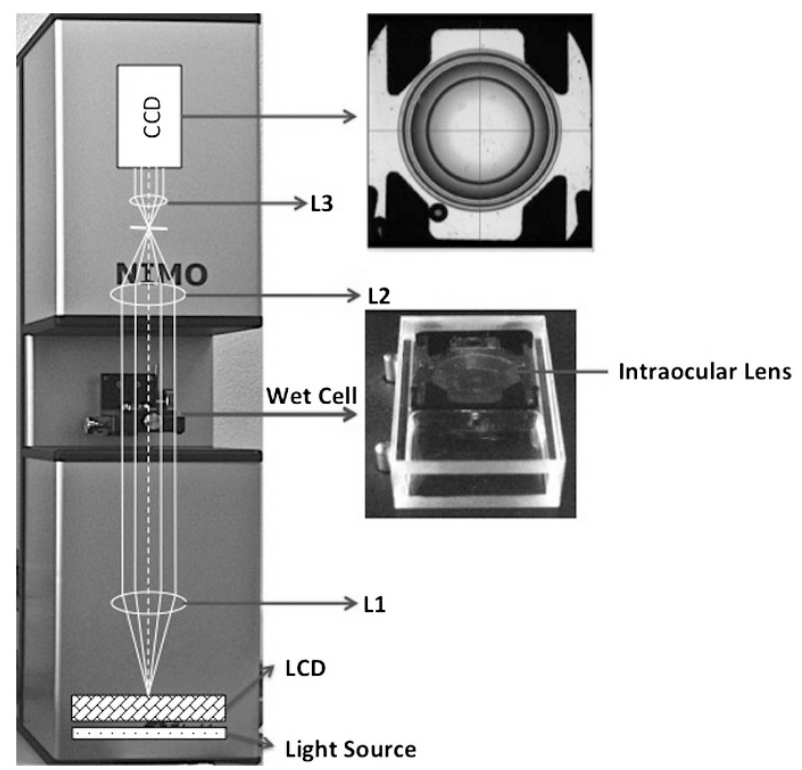

Figure 1 Schematic layout of the NIMO TR1504 (LAMBDA-X). This instrument consists of a cold cathode tube backlight sources that emits green light at $546 \mathrm{~nm}$, incorporating an additional diffuser and a $\pm 10-\mathrm{nm}$ bandwidth filter to the source to homogenize and limit the spectral width of the light beam. A liquid crystal display (LCD) placed at the focal length of the lens L1 that is the lens responsible for collimating the light beam. Two lenses, L2 and L3, which form an image on camera through a telecentric arrangement and a CCD camera with a native pixel resolution of $1396 \times 1340$ pixels corresponding to a spacing of 69 $\mathrm{pixel} / \mathrm{mm}$ or $1761 \mathrm{pixel} / \mathrm{inch}$. The high resolution of the camera is directly related to the high instrument resolution $(36 \mu \mathrm{m})$. The intraocular lens under study is placed in the object plane of the instrument, between lens L1 and L2.

NIMO TR1504. The working principle of this instrument is based on a phase-shifting schlieren techonology. ${ }^{4}$

By combining the principle of schlieren imaging with the phase-shifting method, the NIMO instrument allows the measurement of light beam deviations, which can be used to calculate the wavefront analysis considering the 36 Zernikes coefficients. This technology has been shown to effectively measure in vitro optical quality of intraocular lenses. ${ }^{5}$ The apparatus complies with the International Standard Organization (ISO) 11979-216. ${ }^{6}$ All IOLs were measured while being immersed in a saline solution whose composition was 0.154 milliequivalents per milliliter of $\mathrm{NaCl}$ (Laboratoires Sterop SA, Anderlecht, Belgium). The cuvettes or wet cells used to hold the IOLs and the saline solution in place during the measurements have been verified by means of an interferometer; and were shown to have a power $<0.005 \mathrm{D}$. This additional cross-check on the wet cells was carried out to rule out potential interferences with the measurement. Moreover, accurate power measurements are only possible if the setup has been thoroughly calibrated, which is why the instrument was calibrated for each measurement. 
All measurements were recorded for a $5.0 \mathrm{~mm}$ aperture, as it generally corresponds to the average pupil size of patients who are over 60 years of age with under mesopic conditions. ${ }^{7}$ In this study, Zernikes coefficients values were retained as the average of five measurements.

\section{Image quality parameters}

The IOLs' optical quality was quantified by means of the modulation transfer function (MTF) and the point spread function (PSF). The MTF represents the contrast attenuation induced by an optical system as a function of the spatial frequency or target size. This fall in image contrast, which is sharper for higher spatial frequencies, ${ }^{8}$ can lead to a decline in the optical system's visual performance. ${ }^{9}$

In the current study, the mean one-dimensional MTF was calculated as the average over all orientation of the two-dimensional MTF. Both the MTF and the PSF were computed from the experimental wavefront data using a custom-made MATLAB-based application (Mathworks, Nantick, MA, USA). In order to be able to compare the MTFs yielded by the four IOLs, we took the average modulation value as optical quality metrics. ${ }^{10,11}$ The average modulation has been considered to be the modulation averaged across all frequencies ranging from 0.0 to 100.0 cycles per millimetre; this value has been shown to be proportional to the area under the MTF curve between 0.0 and 120.0 cycles per millimetre.

As it was mentioned before, the image quality of the lenses alone was measured in vitro. Furthermore, to simulate the optical quality that the IOLs could provide after implantation, a whole-eye wavefront aberration map was simulated by adding a corneal aberration profile to the IOLs' aberration profiles, and from these data new MTFs and PSFs were computed. Previous studies have shown that corneal HOAs remain rather stable with age. ${ }^{12-14}$ From those studies, an average corneal pattern could be obtained. To combine (add) this average corneal pattern with each IOL's pattern we used the abovementioned custom-made MATLAB-based application.

The results yielded by the IOLs alone and by the IOL + cornea systems were compared in order to have an estimate of the optical quality that the implanted IOLs could provide.

\section{Results}

Figure 2 presents the HOAs Zernike coefficients for each of the IOLs alone, measured in vitro, as well as the Zernike coefficients corresponding to the averaged cornea. With this figure it is possible to estimate how the Zernike modes would be modified when the corneal aberration coefficients were added to the HOA profile of
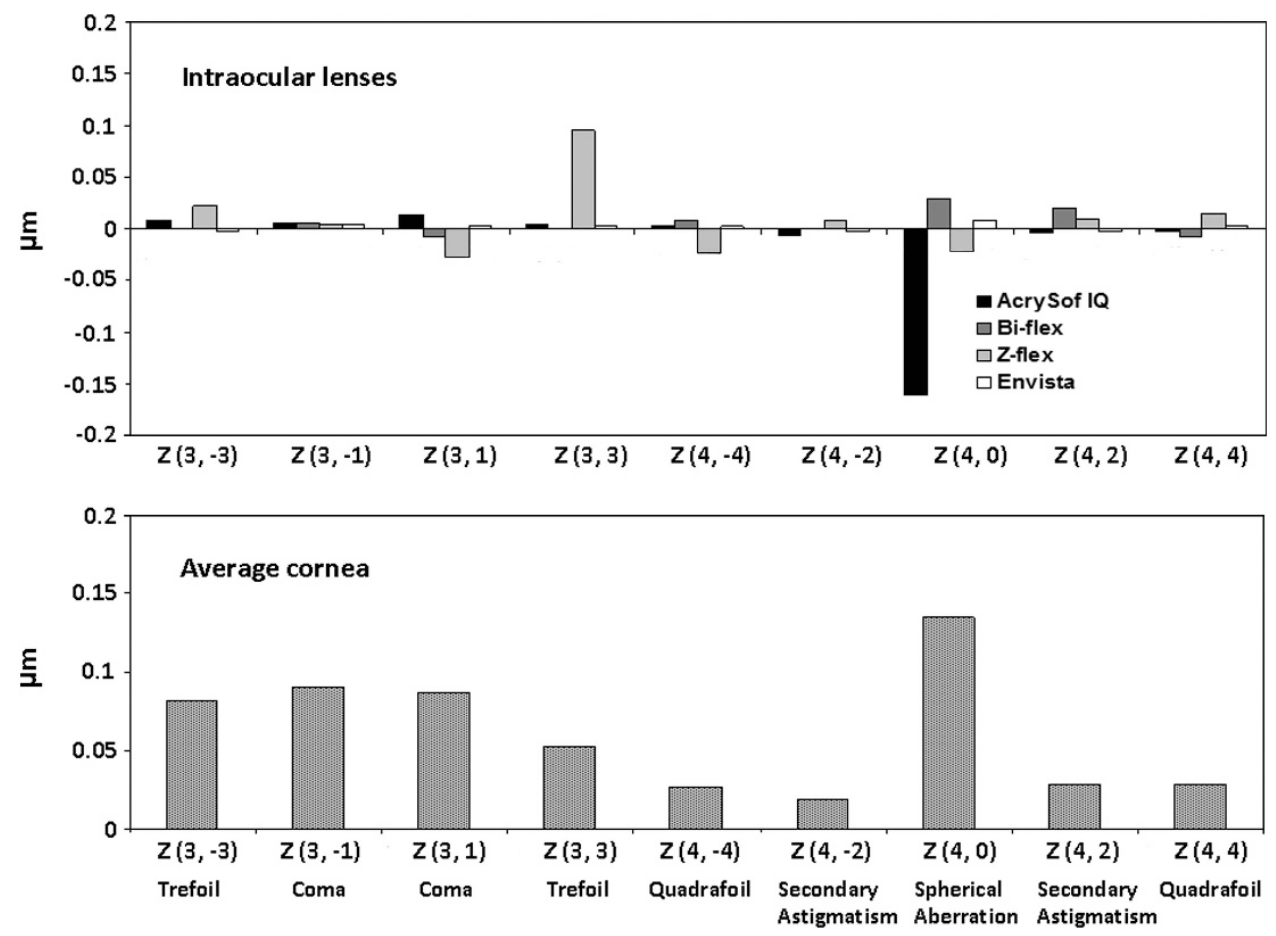

Figure 2 Zernike coefficients of the higher order aberrations corresponding to the intraocular lenses (IOL) alone and the intraocular lenses when an average cornea was added (IOL + cornea system). 
each IOL. In the top of the figure it can be observed that, within all the Zernike modes, the most dramatic differences can be founded for the $\mathrm{Z}(4,0)(\mathrm{SA})$ coefficient if the AcrySof IQ IOL SN60WF is compared to the other three lenses. The figure shows how the AcrySof IQ IOL SN60WF would neutralize the positive SA while the other three would show a positive increment in the SA coefficient when they were combined with the averaged cornea. How this coupling effect would impact on the optical quality of the patients is showed in Figures 3 and 4.

Figures 3 and 4 and show the MTF curves and the PSF for the four IOLs alone and for the four IOL + cornea systems, respectively. From Figure 3 it is possible to observe that the EnVista IOL that provided the best MTF curve when the IOLs were assessed by themselves, without including the effect of corneal aberrations. This is followed by the other 2 aberration-free IOLs. The MTF for the AcrySof IQ IOL SN60WF alone was lower than for the other three IOLs. This is expected because this lens incorporates a negative amount of SA $(-0.20 \mu \mathrm{m}$ for a 6-mm pupil) into its design. However, when the cornea was added (IOL + cornea), the situation reversed completely, since out of the four IOLs under study it was the AcrySof IQ IOL SN60WF that yielded the best results: for all spatial frequencies the MTF values obtained for the AcrySof IQ IOL SN60WF + cornea system were higher than for the other three systems. It is also interesting to observe that the AcrySof IQ IOL SN60WF
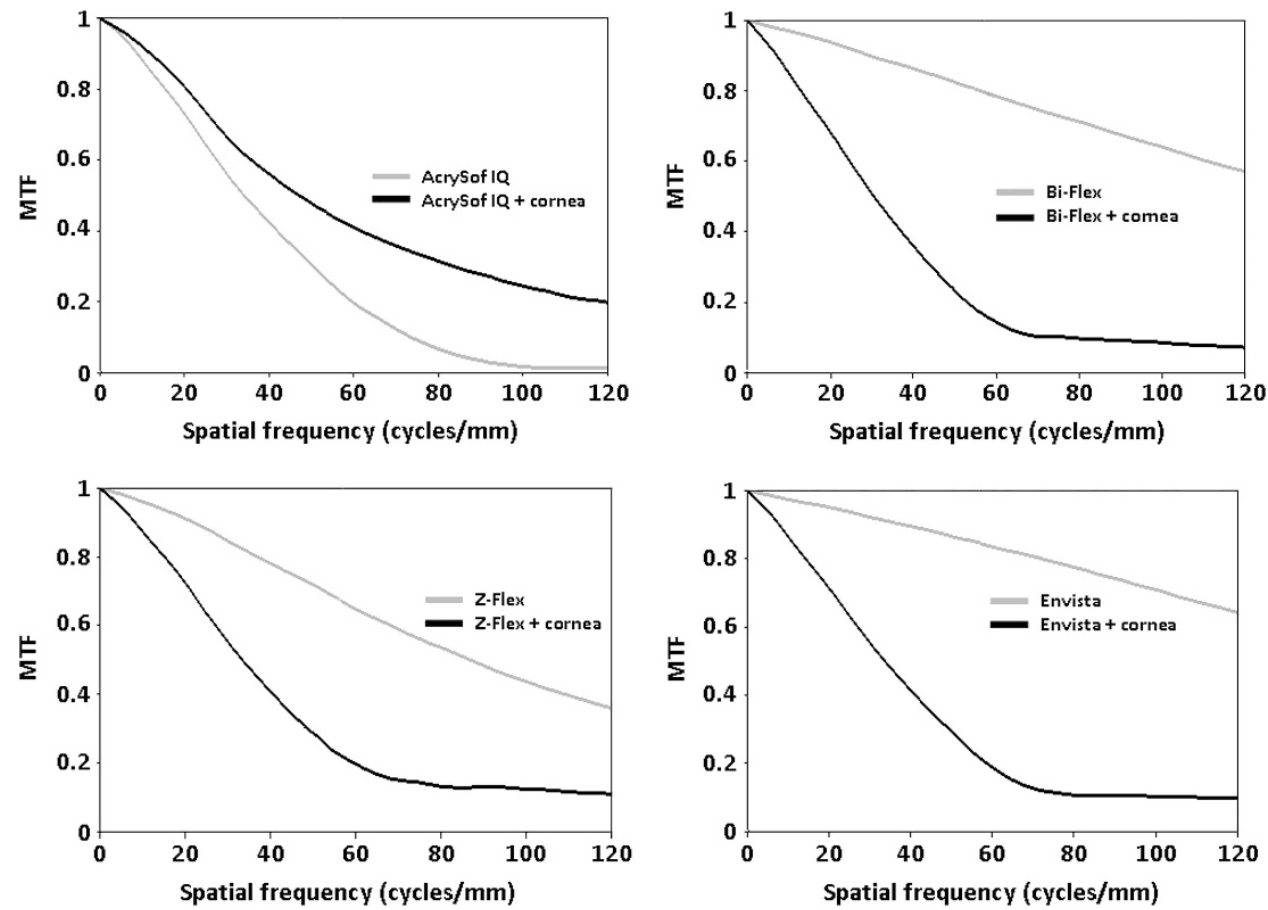

was the only lens whose MTF curve improved upon addition of the cornea, which is comparable to the postimplatation status of the IOL inside of the eye.

Table 1 presents the average modulation values for the four IOLs under study in both scenarios (IOL by itself or combined with the average corneal profile). The data reveal that the EnVista IOL offered the best results when the lenses were considered without the cornea, whereas when the IOLs were considered as part of an IOL + cornea system, it was the AcrySof IQ IOL SN60WF that yielded the best results out of the four IOLs.

\section{Discussion}

In the current study we have evaluated a new aberrationfree IOL that it has been launched into the market. This new lens is the EnVista IOL and in the present study it has been experimentally compared by means of in-vitro measurements of their optical quality with three other aspheric monofocal IOLs: AcrySof IQ IOL SN60WF, Bi-Flex 1.8 677AB and Z-Flex HB 860FAB. The EnVista, the Z-Flex and the Bi-Flex IOLs have all neutral aspheric designs - that is, they are IOLs with no intrinsic SA (aberration-free)—, whereas the AcrySof IQ IOL SN60WF is an aberration-correcting aspheric IOL that has been designed to have a negative amount of SA in an attempt to partially compensate for the positive ocular SA that the eye normally has once the lens has been extracted. Optical quality metrics (MTF, PSF) were computed both

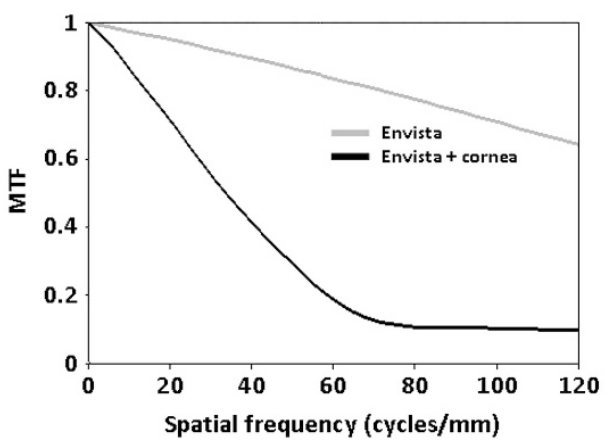

Figure 3 Modulation transfer function curves of the four intraocular lenses (IOL) measured in vitro, both alone (without a cornea) and when the average corneal pattern was added (IOL + cornea system). 

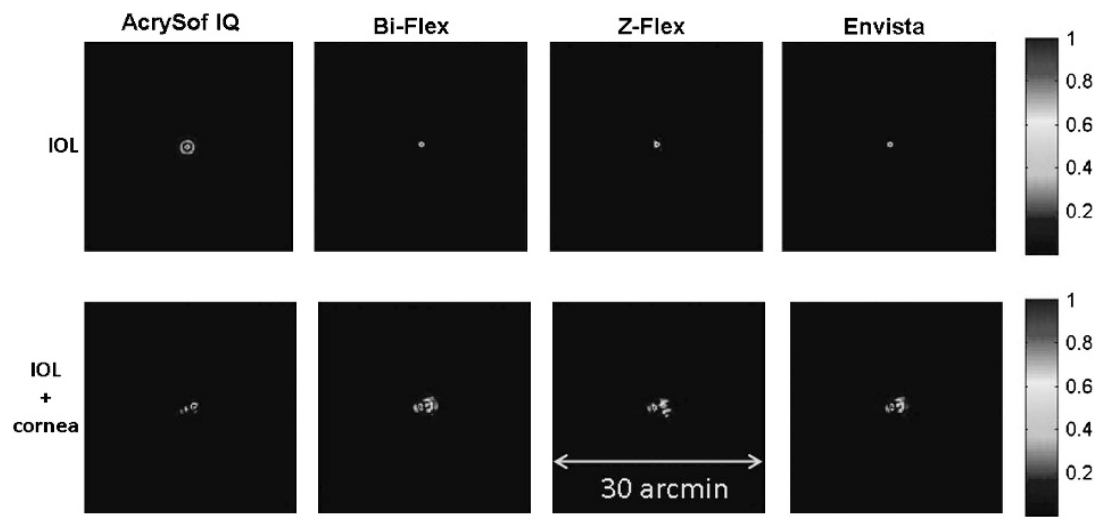

Figure 4 Point spread function representation of the four intraocular lenses (IOL) measured in vitro, both alone (without a cornea) and when the average corneal pattern was added (IOL + cornea system).

Table 1 Average modulation values for the four IOLs under study, both alone (without a cornea) and when the average corneal pattern was added (IOL + cornea system), for a $5.0 \mathrm{~mm}$ aperture

\begin{tabular}{lcccc}
\hline & AcrySof IQ & Bi-flex & Z-Flex & Envista \\
\hline IOL & 39.5 & 82.5 & 72.3 & 86.4 \\
IOL + cornea & 56.0 & 36.4 & 40.2 & 39.3 \\
\hline
\end{tabular}

for the IOLs alone as well as for a hypothetical optical system comprising each IOL plus an average cornea, whose aberration data map was taken from the literature. ${ }^{12-14}$ This latter scenario was included in order to estimate the performance of the IOL once it is implanted in the aphakic eye.

Figures 3 and 4 show the MTF curves and the PSF, respectively. These figures present the results of the IOLs measured in vitro and the IOLs when the average corneal HOAs were added. Both the MTF curves and the PSF suggest that when the IOLs are measured without cornea, the aberration-free IOLs performed better than the aberration-correcting IOL. However, when the HOAs of the cornea were added to the aberration profile of the IOLs, the aberration-correcting IOL was the only IOL that improves its optical quality when compared with the situation without cornea. All these results clearly show the impact of SA upon the optical performance of an IOL: one can see that if the cornea is not taken into account, the aberration-free IOLs present the lowest amounts of SA and higher MTF and PSF values. Conversely, when the cornea was added, the aberration-correcting IOL present the lowest amount of SA and the best results. In terms of visual quality, different studies have reported the importance of SA, showing that an increase in the total amount of the eye's SA results in an increase of the total amount of HOAs, which, in turn, decrease the patients' visual function. ${ }^{15,16}$ The dramatic changes in the results observed when the corneal profile was added to the IOL profile, remark the importance of including a corneal profile to the IOL profile in order to represent, as near as possible to a 'real life' scenario, the potential optical performance of an IOL once it is implanted in the aphakic eye.

In the current study we have quantified the optical performance by means of the MTF. In relation to visual quality, a decrease in the MTF entails variations in image contrast that could reduce an optical system's image quality, thus affecting the patients' visual performance. ${ }^{9}$ Moreover, Felipe et $a l^{17}$ performed a study to determinate whether there is a correlation between IOL optics and visual parameters. In order to do that, the authors analysed the optical quality of the IOL by average modulation parameter and the patient's visual quality by the visual acuity. The authors found that the visual acuity varied 0.24 decimal units per 10 units of average modulation variation under mesopic conditions. Table 1 shows the average modulation values of the four IOLs for a 5-mm aperture (it generally corresponds to the average pupil size that patients over 60 years of age have under mesopic conditions. ${ }^{7}$ ). In this study, when the cornea was added to the IOLs, it was the AcrySof IQ IOL SN60WF (aberration-correcting) that provided the best results, showing a substantial difference with the other three IOLs (see Table 1). It is important to take into account these results and the differences across the lenses due to the reported impact that they would have on the visual quality of the patients. ${ }^{17}$ For example, considering the study of Felipe et al, ${ }^{17}$ the average modulation values obtained in the current study could be equivalent to visual acuity values under mesopic conditions about 20/16 for IQ, 20/20 for Z-Flex and 20/25 for Bi-Flex and EnVista. Therefore, the correction of the SA under the mentioned conditions improves the optical quality of the $\mathrm{IOL}+$ cornea system and consequently, it is possible to suggest that the visual quality of the patients would also be improved in regular tasks performed in mesopic 
conditions such night-driving. These outcomes have showed the importance of correcting the SA of the $\mathrm{IOL}+$ cornea system in order to attain an optimum visual performance.

It should be also noted that we used an average corneal profile that is a theoretical representation of the corneal profile of the population. This allows to estimates the optical performance when the IOLs profile is added to the averaged cornea. Nevertheless, the corneal profile could slightly vary within normal individuals but largely within patients that have undergone corneal refractive surgeries. ${ }^{18-21}$ For these cases, due to the HOA profiles of the IOL are also showed and the surgeons could assess the corneal profile of each patient, it would be possible to estimate which is the best IOL to each individual case.

At the same time, it is also important to bear in mind that various factors related to the surgical implantation of the IOLs could also have an impact upon the patient's visual quality. One of the most important factors is the required accuracy of the centration of the lens. ${ }^{18-24}$ Decentration of an IOL can lead to other common complication: tilt. In fact, tilt and decentration of the implanted IOLs could limit or even cancel out the theoretical advantages of aspheric IOLs. ${ }^{23-27}$

In summary, the results of the current study show that the EnVista IOL studied provides a good optical performance, however, the optical quality results for this IOL were worse than for the aberration-correcting IOL. Hence, it seems that it would be interesting to consider other designs for this new IOL with other degrees of SA in order to optimize its results in a wider spectrum of patients.

\section{Summary}

\section{What was known before}

- In order to improve their product offering, the specialized industry of intraocular lenses (IOLs) has launched periodic innovations into the market. Two of the most important areas of innovation are related to the role of higher-order aberrations (HOAs), being the spherical aberration (SA) the most important one, and the materials from which the IOLs are manufactured. Recently, it has been launched into the market a new IOL manufactured from a novel hydrophobic acrylic material with an aberration-free design; this new lens is the EnVista. The optical quality related to the visual quality of patients with these new IOL had not been studied.

\section{What this study adds}

- The in vitro optical performance of the EnVista IOL was good, but it decreases substantially in a whole-eye scenario, when the wavefront profile of an average cornea is added. Other designs with different degrees of SA should be considered for this new IOL in order to surpass these results.

\section{Conflict of interest}

The authors declare no conflict of interest.

\section{Acknowledgements}

This research was supported in part by a Research Grant (\#SAF2009-13342\#) of the Ministerio de Ciencia e Innovación (Ministry of Science and Innovation) awarded to Robert Montés-Micó.

\section{References}

1 Olson RJ, Crandall AS. Prospective randomized comparison of phacoemulsification cataract surgery with a $3.2-\mathrm{mm} v s$ a 5.5-mm sutureless incision. Am J Ophthalmol 1998; 125: 612-620.

2 Oshika T, Nagahara K, Yaguchi S, Emi K, Takenaka H, Tsuboi $\mathrm{S}$ et al. Three year prospective, randomized evaluation of intraocular lens implantation through 3.2 and $5.5 \mathrm{~mm}$ incisions. J Cataract Refract Surg 1998; 24: 509-514.

3 Zheng L, Merriam JC, Zaider M. Astigmatism and visual recovery after 'large incision' extracapsular cataract surgery and 'small' incisions for phakoemulsification. Trans Am Ophthalmol Soc 1997; 95: 387-415.

4 Joannes L, Dubois F, Legros JC. Phase-shifting schlieren: highresolution quantitative schlieren that uses the phaseshifting technique principle. Appl Opt 2003; 42(25): 5046-5053.

5 Pérez-Vives C, Ferrer-Blasco T, Madrid-Costa D, García-Lázaro S, Montés-Micó R. Optical quality comparison of conventional and hole-visian implantable collamer lens at different degrees of decentering. Am J Ophthalmol 2013; 156(1): 69-76.

6 International Organization for Standardization. Ophthalmic Implants-Intraocular Lenses_Part 2: Optical Properties and Test Methods. ISO: Geneva, Switzerland, 1999, ISO 11979-2 $1-27$.

7 Winn B, Whitaker D, Elliott DB, Phillips NJ. Factors affecting light-adapted pupil size in normal human subjects. Invest Ophthalmol Vis Sci 1994; 35: 1132-1137.

8 Gatinel D, Pagnoulle C, Houbrechts Y, Gobin L. Design and qualification of a diffractive trifocal optical profile for intraocular lenses. J Cataract Refract Surg 2011; 37: 2060-2067.

9 Artigas JM, Menezo JL, Peris C, Felipe A, Díaz-Llopis M. Image quality with multifocal intraocular lenses and the effect of pupil size; comparison of refractive and hybrid refractive-diffractive designs. J Cataract Refract Surg 2007; 33: 2111-2117.

10 Artigas JM, Peris C, Felipe A, Menezo JL, Sánchez-Cortina I, López-Gil N. Modulation transfer function: rigid versus foldable phakic intraocular lenses. J Cataract Refract Surg 2009; 35: 747-752.

11 Marsack JD, Thibos LN, Applegate RA. Metrics of optical quality derived from wave aberration predict visual performance. J Vis 2004; 4: 322-328.

12 Oshika T, Klyce SD, Applegate RA, Howland HC. Changes in corneal wavefront aberrations with aging. Invest Ophthalmol Vis Sci 1999; 40: 1351-1355.

13 Guirao A, Redondo M, Artal P. Optical aberrations of the human cornea as a function of age. J Opt Soc Am A Opt Image Sci Vis 2000; 17: 1697-1702. 
14 Wang L, Dai E, Koch DD, Nathoo A. Optical aberrations of the human anterior cornea. J Cataract Refract Surg 2003; 29: 1514-1521.

15 Li J, Xiong Y, Wang N, Li S, Dai Y, Xue L et al. Effects of spherical aberration on visual acuity at different contrasts. J Cataract Refract Surg 2009; 35: 1389-1395.

16 Barbero S, Marcos S, Jimenez-Alfaro I. Optical aberrations of intraocular lenses measured in vivo and in vitro. J Opt Soc Am A Opt Image Sci Vis 2003; 20: 1841-1851.

17 Felipe A, Pastor F, Artigas JM, Diez-Ajenjo A, Gené A, Menezo JL. Correlation between optics quality of multifocal intraocular lenses and visual acuity: tolerance to modulation transfer function decay. J Cataract Refract Surg 2010; 36: 557-562.

18 Madrid-Costa D, Ruiz-Alcocer J, Pérez-Vives C, Ferrer-Blasco T, López-Gil N, Montés-Micó R. Visual simulation through different intraocular lenses using adaptive optics: effect of tilt and decentration. J Cataract Refract Surg 2012; 38: 947-958.

19 Madrid-Costa D, Pérez-Vives C, Ruiz-Alcocer J, Albarrán-Diego C, Montés-Micó R. Visual simulation through different intraocular lenses in patients with previous myopic corneal ablation using adaptive optics: effect of tilt and decentration. J Cataract Refract Surg 2012; 38: 774-786.

20 Ruiz-Alcocer J, Pérez-Vives C, Madrid-Costa D, López-Gil N, Montés-Micó R. Effect of simulated IOL tilt and decentration on spherical aberration after hyperopic
LASIK for different intraocular lenses. I Refract Surg 2012; 28: 327-334.

21 Madrid-Costa D, Ruiz-Alcocer J, García-Lázaro S, Albarrán-Diego C, Montés-Micó R. Visual performance of the Akreos Adapt AO intraocular lens in patients with different corneal profiles measured with an adaptive optics visual simulator. Br J Ophthalmol 2012; 96: 1099-1103.

22 Montés-Micó R, López-Gil N, Pérez-Vives C, Bonaque S, Ferrer-Blasco T. In vitro optical performance of nonrotational symmetric and refractive-diffractive aspheric multifocal intraocular lenses: Impact of tilt and decentration. J Cataract Refract Surg 2012; 38: 1576-1582.

23 Gimbel HV, Condon GP, Kohnen T, Olson RJ, Halkiadakis I. Late in-the-bag intraocular lens dislocation: incidence, prevention, and management. J Cataract Refract Surg 2005; 31: 2193-2204.

24 Carlson AN, Stewart WC, Tso PC. Intraocular lens complications requiring removal or exchange. Surv Ophthalmol 1998; 42: 417-440.

25 Atchison DA. Design of aspheric intraocular lenses. Ophthalmic Physiol Opt 1991; 11: 137-146.

26 Altamann GE, Nichamin LD, Lane SS, Pepose JS. Optical performance of 3 intraocular lens designs in the presence of decentration. J Cataract Refract Surg 2005; 31: 574-585.

27 Montés-Micó R, Ferrer-Blasco T, Cerviño A. Analysis of the possible benefits of aspheric intraocular lenses: review of the literature. J Cataract Refract Surg 2009; 35: 172-181. 